\title{
Assessment of representativity of the PETALE experiments for validation of Swiss LWRs ex-core dosimetry calculations
}

\author{
Alexander Vasiliev ${ }^{1, *}$, Marco Pecchia ${ }^{1}$, Dimitri Rochman $^{1}$, Gregory Perret ${ }^{1}$, Hakim Ferroukhi ${ }^{1}$, \\ Axel Laureau ${ }^{2}$, Vincent Lamirand ${ }^{2}$, and Andreas Pautz ${ }^{1,2}$ \\ ${ }^{1}$ Laboratory for Reactor Physics and Thermal-Hydraulics (LRT), \\ Paul Scherrer Institute (PSI), Forschungsstrasse 111, 5232 Villigen, Switzerland \\ ${ }^{2}$ Laboratory for Reactor Physics and Systems Behaviour (LRS), \\ École Polytechnique Fédérale de Lausanne (EPFL), 1015 Lausanne, Switzerland
}

\begin{abstract}
The international experimental program PETALE will be carried out at the CROCUS research reactor of EPFL. The program aims at measuring neutron penetration in slabs made of materials composing typical LWR reactor pressure vessel. The measurements will be used for code and nuclear data validation and for the evaluation of the reflecting properties of these materials. In this paper the representativity of the PETALE experiments is assessed with respect to operational LWR reactors dosimetry and activation evaluations using the Paul Scherrer Institute (PSI) in-house tool NUSS. The NUSS tool allows the stochastic sampling of nuclear data using covariance matrices available in modern nuclear data libraries and the subsequent running of a Monte Carlo code with the modified data files. The representativity can then be assessed based on the Pearson correlation coefficients. The ultimate goal of the work is first of all to assess if the planned PETALE measurements could be applicable beyond their primary purpose and serve for extending the PSI validation database for LWR reactor dosimetry evaluations. Secondly, provided that the PETALE measurements are found useful for the task above, the information on the correlations between the PETALE neutron detectors' responses and the reactor dosimetry quantities of interest, shall be presented and discussed.
\end{abstract}

\section{Introduction}

The fast neutron fluence (FNF) is a conventional parameter for analysis in the LWR dosimetry. The number of publicly open evaluated benchmark experiments applicable for validation of the calculation methodologies for FNF assessment is nevertheless limited and therefore every appropriate benchmark or available experimental data from operational power reactors are practically very valuable.

In this work the representativity of the PETALE experimental program, which is currently under development at the CROCUS research reactor of EPFL, is assessed. CROCUS is an experimental zero-power, uranium-fuelled and water-moderated reactor. CROCUS is licensed for operating at a maximum power of $100 \mathrm{~W}$, i.e. up to $2.510^{9}$ $\left[\mathrm{n} / \mathrm{cm}^{2} s\right]$ total neutron flux at the core centre.

The original purpose of the program is to measure the neutron transport through materials typical for "heavy reflectors" as found, for example, in the EPR reactor design and thus to provide validation data for the selected materials cross-sections and reflector properties. However, the PETALE setup can also be useful for providing experimental data applicable for validation of calculation methodologies for typical LWR reactor dosimetry applications, like the one being developed and validated at PSI [1], [2], [3]. Thus, a pertinent question which can

*e-mail: alexander.vasiliev@psi.ch be posed is: how representative are the planned PETALE measurements for the validation of FNF predictions in LWR structures?

From the viewpoint of validation of nuclear data libraries for FNF simulations, such question can be investigated with the help of the correlation analysis as illustrated in the examples below. In order to assess the applicability of the foreseen PETALE experimental data in comparison with traditional LWR dosimetry measurements, two publicly open benchmarks are included into the analysis: H.B. Robinson-2 RPV Dosimetry Benchmark and the PCA-Replica $\left(\mathrm{H}_{2} \mathrm{O} / \mathrm{Fe}\right)$ Shielding Benchmark from the OECD/NEA Radiation shielding and dosimetry experiments database SINBAD (see https://www.oecdnea.org/science/wprs/shielding/sinbad/).

The neutron transport simulations for the given study were performed with the help of the Monte Carlo NParticle $₫$ MCNP $®$ Software (see https://mcnp.lanl.gov/) and for some cases with MCNPX-2.4.0 [4] and SERPENT 2 code v.2.1.30 (see http://montecarlo.vtt.fi/), in conjunction with the nuclear data based on the ENDF/BVII.1 library [5]. The nuclear data uncertainties propagation and the calculation parameters correlation analysis were done with the help of the PSI in-house tool NUSS, which allows the stochastic sampling of the ACEformatted nuclear data files using the covariance matrices available in the modern nuclear data libraries. 


\section{PETALE Experimental Setup at EPFL/CROCUS Research Reactor}

The CROCUS reactor core is approximately $100 \mathrm{~cm}$ in height and about $60 \mathrm{~cm}$ in diameter. The core consists of two fuel zones with square lattices of different pitches. The core inner part consists of $336 \mathrm{UO}_{2}$ rods with 1.8 wt.\% enriched and the outer part consists of 172 metal uranium rods with $0.95 \mathrm{wt}$ \% enrichment. The fuel rods cladding is made of aluminium. The fuel rods are assembled with two aluminium grid plates which have a $0.5-\mathrm{mm}$ cadmium layer to limit axial neutron leakage.

The PETALE experimental setup is designed to represent the 'heavy reflector' surrounding LWR cores, as is the case for the EPR. In particular, eight metallic reflector plates, each 2-cm thick, will be installed next to the CROCUS reactor core. A set of separate configurations will be assembled using eight iron, chromium, nickel, or stainless steel plates in a watertight box. Neutron dosimetry detectors will be installed around and within the reflector plates.

After irradiation, the dosimeters' activities will be measured using high-purity germanium (HPGe) gamma spectrometers to be compared with calculation results. The targeted part of the reflector materials' nuclear data for consequent validation studies is in the $\mathrm{MeV}$ energy range. The fast flux $(>0.1 \mathrm{MeV})$ is around $10^{8}\left[\mathrm{n} / \mathrm{cm}^{2} s\right]$ in the reflector area close to CROCUS. In addition to the reflector transmission experiments, reflector reactivity worth estimates are also planned. A sketch of the experimental configuration is illustrated in Fig. 1 and more detailed information can be found in [6], [7] [8].

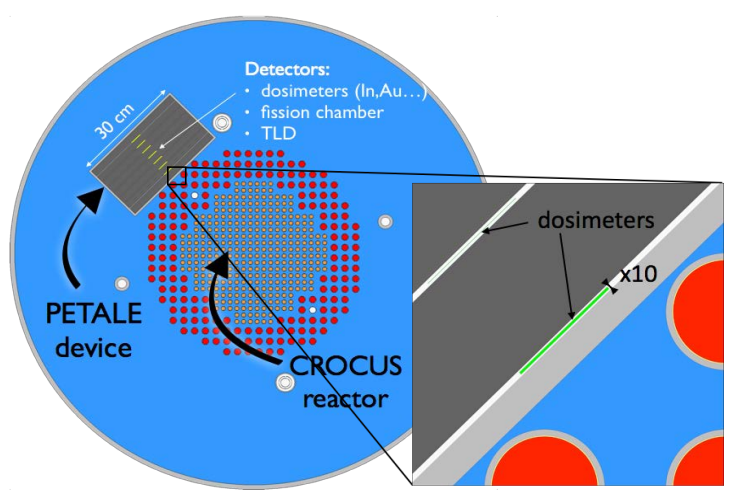

Figure 1. Illustration of the PETALE experimental set up.

\section{NUSS: Nuclear Data Stochastic Sampling with ACE files}

The NUSS tool developed at PSI allows to randomly sample nuclear data in the form of ACE formatted files. NUSS currently treat all nuclear data (ND) distributions as multivariate normal (MVN) distributions. This limitation is adequate given the lack of knowledge on the underlying nuclear data distributions. Details on the NUSS methodology, its validation and application studies can be found in [9], [10], [11], [12], [13].
The inherent power of the stochastic sampling approach is the capability to propagate the underlying input data (here the nuclear data) uncertainties up to an arbitrary output calculation parameter. In that respect, NUSS is equally applicable to any type of neutron transport simulations, would it be a criticality safety problem or dosimetry and activation application. Another valuable feature of the stochastic sampling is the ease with which one can perform the correlation analysis, should it be between the output and input parameters of a given simulation (see, e.g., [11]) or between the output parameters of different simulation models (as in this work). Similar studies can theoretically be done with deterministic approaches, although the practical realization is normally limited by the class of the output parameters to which the (first-order) sensitivity coefficients can be conveniently obtained [14].

Up to now, NUSS has been tested for different types of systems with different quantities of interest and for different tasks, namely for nuclear data uncertainty quantification (UQ) and parameter correlation studies using the Pearson correlation coefficient (PCC), as well as for a variance-based global sensitivity analysis (GSA) [11]. PCC computed in correlation studies can be interpreted as the measure of similarity between the tested systems, or, similarly, as a representativity measure of a benchmark model with respect to a certain application system. Up to now, the correlation studies with NUSS have covered both Criticality Safety and Dosimetry applications, as can be found in [15], [16], [17].

\section{Details of the present study}

The present study is focused on the investigation of representativity of the expected PETALE experimental data for validation of LWR FNF predictions. In order to facilitate the interpretation of the calculation results, the assessment of the well-known benchmark experiments was added to the scope of the study, namely the H.B. Robinson-2 RPV Dosimetry Benchmark (HBR-2) and the PCA-Replica $\left(\mathrm{H}_{2} \mathrm{O} / \mathrm{Fe}\right)$ Shielding Benchmark are considered in this work. Both selected benchmark experiments were simulated with the MCNP software. In particular, the PCA-Replica model was calculated with MCNP-6.1.1 and the HBR-2 model was calculated with MCNPX-2.4.0. The utilized model of the HBR-2 benchmark is that used in [18] ${ }^{1}$ and its schematic representation is given in Fig. 2. The previously obtained C/E results and modelling details for the HBR2 benchmark simulation with MCNPX-2.4.0 were reported in [18], [19].

The PCA-Replica model was borrowed from the recent benchmark updated specifications [20] . The calculations were done using ENDF/B-VII.1 library for the neutron transport related ND and IRDFF-1.05 for the dosimetry reaction cross-sections.

As presented in full details in [8], the CROCUS/PETALE setup was modelled with the SERPENT 2. To save computation time, the original PETALE design has been slightly modified. In particular, the volumes of

\footnotetext{
1'method c' in Section 4.2 of [18]
} 


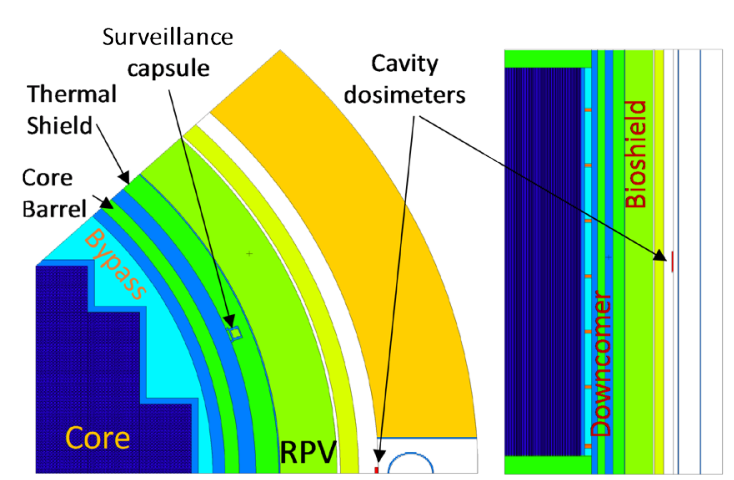

Figure 2. Illustration of the H.B. Robinson-II RPV Dosimetry Benchmark, as modelled with MCNPX.

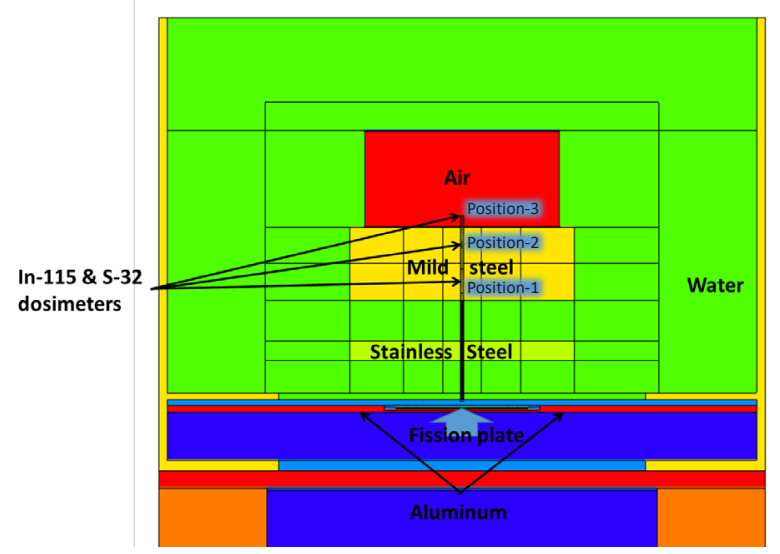

Figure 3. Illustration on of the PCA-Replica MCNP calculation model.

the dosimeters have been artificially increased to improve the statistical precision of the SERPENT calculations, as illustrated in Fig. 4. Such approximation will not be used for the final experiment planning and analysis, however it shall be sufficient for the nuclear data uncertainties propagation study presented here. This study is also restricted to the case of the iron reflector plates.

For the PETALE model calculations with SERPENT 2, the ENDF/B-VII.1 library was used as the source of the ND for both the neutron transport and dosimetry reactions.

Finally, in addition to the above benchmarks and PETALE models, a model of a Swiss PWR was also calculated with the same ACE random files based on the ENDF/B-VII.1 library, to represent a practical application case. As demonstrated later, the overall set of calculations allows deriving correlation coefficients between all calculation outputs. The correlations between the PWR RPV FNF and the dosimeters in PETALE are of primary interest.

One can notice that no BWR model has been yet included in the analysis. Nevertheless, according to the results presented in [15], PWR and BWR reactor vessel dosimetry calculations are highly correlated and therefore the findings obtained in this work for the PWR cases shall be also indirectly valid for BWRs. Explicit analysis of cor-

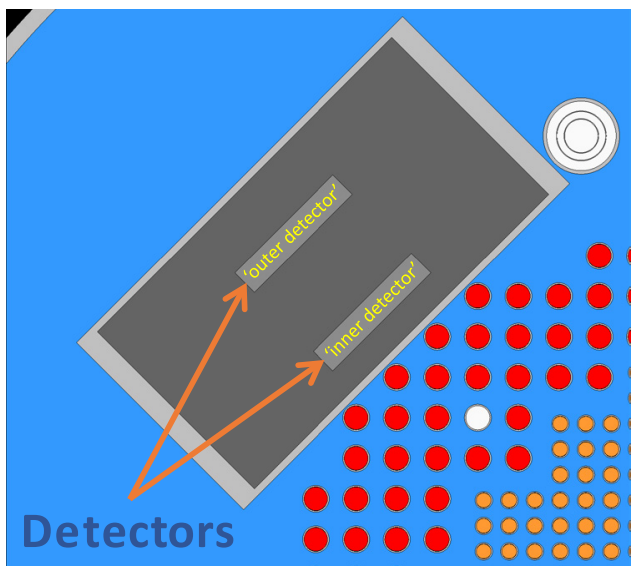

Figure 4. Simplified detector simulation with SERPENT 2 PETALE model implemented in this work.

relations between the PETALE and BWR simulations with NUSS is currently planned for the near future.

Regarding NUSS, the list of perturbed ND included about 50 isotopes of the fuel, coolant and structural materials. The perturbed reactions included $(n, n),(n, n /),(n, 2 n)$ and $(\mathrm{n}, \gamma)$ cross-sections for non-actinides and in addition $(\mathrm{n}, \mathrm{f})$, neutron multiplicity $\overline{v_{p}}$ and fission neutron spectra $\chi_{p}$ for actinides. Thus, the dimension of the randomly sampled input space was about 50 isotopes $\cdot 5$ reactions $\cdot 187$ energy bins of covariance matrices, $\approx 50,000$.

It is important to note that only the ND affecting the neutron transport (not the dosimeters reaction rates) were considered in the simulations with NUSS. In total about 250 calculations with random ACE files were realized for each calculation model. The lists of the dosimetry reactions analysed in the HBR-2 and PCA-Replica benchmarks were, respectively: ${ }^{237} \mathrm{~Np}(\mathrm{n}, \mathrm{f}),{ }^{238} \mathrm{U}(\mathrm{n}, \mathrm{f}),{ }^{58} \mathrm{Ni}(\mathrm{n}, \mathrm{p})$, ${ }^{54} \mathrm{Fe}(\mathrm{n}, \mathrm{p}),{ }^{46} \mathrm{Ti}(\mathrm{n}, \mathrm{p}),{ }^{63} \mathrm{Cu}(\mathrm{n}, \alpha)$ with the nominal detector thresholds (as accepted in US NRC, 2001) in MeV: 0.69, $1.45,2.05,2.32,3.76,4.65$ and ${ }^{115} \operatorname{In}(n, n),{ }^{32} \mathrm{~S}(\mathrm{n}, \mathrm{p})$ with the thresholds in MeV: 0.35 and 1.00 .

The considered PETALE dosimetry reactions and the corresponding ranges of lethargy-averaged energies of the interacting neutrons were: ${ }^{103} \mathrm{Rh}(\mathrm{n}, \mathrm{n} \prime),{ }^{115} \operatorname{In}(\mathrm{n}, \mathrm{n} /)$ : $1-2$ $\mathrm{MeV} ;{ }^{58} \mathrm{Ni}(\mathrm{n}, \mathrm{p}),{ }^{54} \mathrm{Fe}(\mathrm{n}, \mathrm{p}): 2-5 \mathrm{MeV}$.

The PCA-Replica benchmark has been analysed using the MCNP model available from the benchmark specifications. The obtained validation results were quite good: the $|\mathrm{C} / \mathrm{E}-1|$ values laid within $5 \%$.

\section{Calculation results}

The results obtained with the MCNP and SERPENT calculations for the considered models and using randomly sampled ACE nuclear data files are demonstrated in Fig. 5. It shall be highlighted that for the given study Monte Carlo calculations with a limited number of particles were performed. However, the results look sufficiently precise for the assessment of representativity of the planned PETALE dosimetry measurements for validation of LWR FNF calculation methodology and employed nuclear data 
libraries. Fig. 5 was prepared with the help of the "PerformanceAnalytics" package for R software environment (see https://cran.r-project.org/ for details).
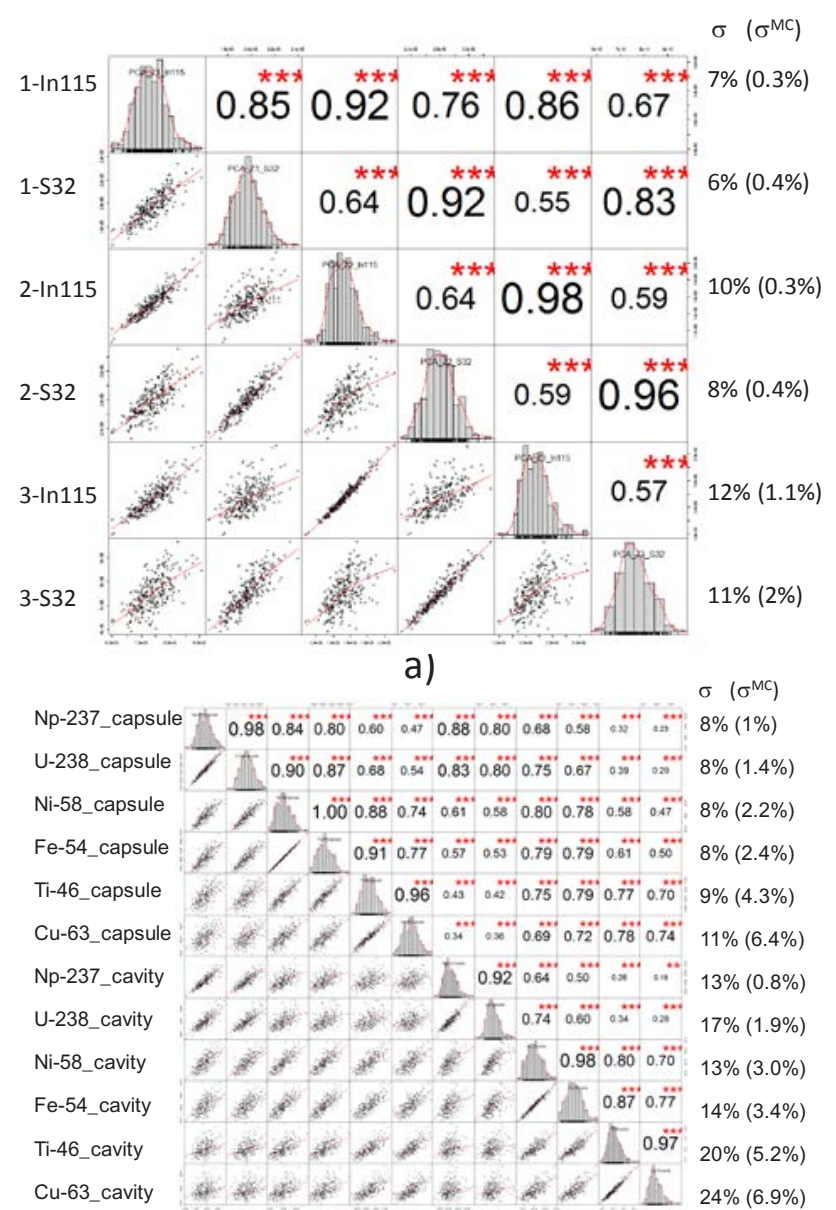

b)

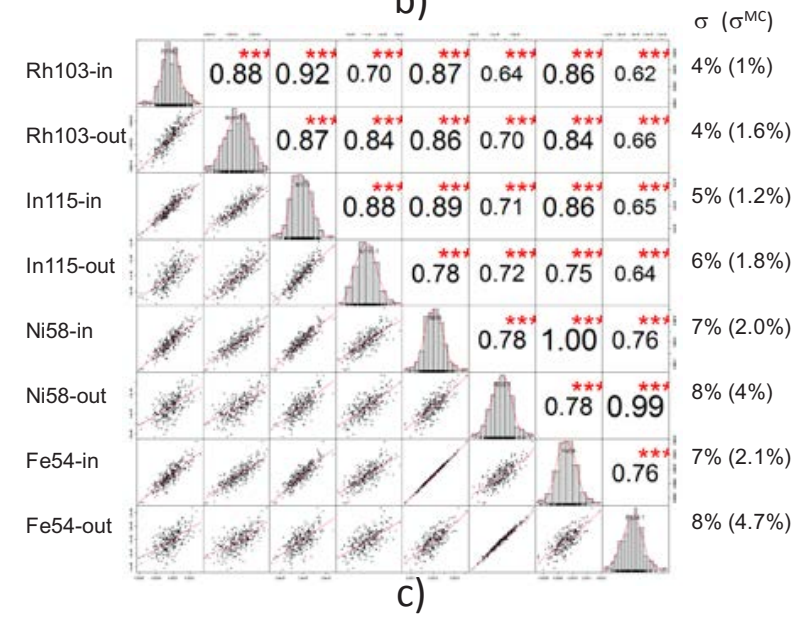

Figure 5. Variance and correlation analysis results for a) PCAReplica; b) HBR2 and c) PETALE/CROCUS models; labels "in" and "out" stand for the inner and outer detectors, as illustrated in Fig. 4.

The standard deviations reported on the right side of the graphs include both the ND related component and the Monte Carlo stochastic uncertainty component. The latter are also reported separately and for certain dosimeters can actually be rather high. However, the Monte Carlo calculation uncertainties are nevertheless much smaller as compared to the total uncertainties and therefore the dominant contributors to the reported standard deviations are the ND uncertainties (note that the uncertainties shall be combined as a sum of variances, or squared standard deviations).

Nevertheless, the PCC estimations can be underpredicted when a noticeable contribution from the Monte Carlo uncertainties is present in the simulation results. In [21] it was demonstrated how the directly obtained correlation coefficients can be effectively corrected with account for the Monte Carlo uncertainty and how the corrected correlation coefficients are higher than the uncorrected ones. Such method will be applied in the follow up studies at PSI with the models considered in this paper. However, in many cases even the uncorrected correlation coefficients are sufficiently high to draw conclusions on the similarity between the considered systems and calculation quantities. Furthermore, all obtained correlation data look physically meaningful which confirms that the overall data processing was done correctly.

An independent ND UQ study has been performed at EPFL and the results can be found in [8]. It is interesting to notice that the ND uncertainty results estimated at EPFL typically grow when the dosimeter is placed between reflector plates farther away from the reactor core. However, the present results obtained with NUSS do not show such strong tendency. The reason can be related to the differences in the simulation approaches, noting that the EPFL ND UQ study was aiming at the optimization of the PETALE experiment design, which required specific modelling assumptions. While at EPFL only the uncertainties of the reflector materials isotopes were considered, together with the uncertainties of the dosimeter reactions, in the present study the nuclear data of the fuel and coolant isotopes were also included into the analysis. This shall moderate the influence of the reflector materials uncertainties and therefore decrease the dependence of the detectors reaction rates uncertainties on the reflector material thickness (which is 'seen' by the neutrons traveling from the core through the reflector plates and up to the detector). Note also that in the EPFL study, the authors were mainly concerned with the ratio of activities delivered by dosimeters located after different thickness of materials. This ratio is far less sensitive to the uncertainty on the nuclear data of the reactor fuel and coolant and this is why their impact was not considered in [8].

In the last step of the analysis, the correlation coefficients between the considered dosimeters of the benchmarks or of the PETALE models and the FNF values evaluated for a Swiss PWR model are reported in Table 1. The FNF results correspond to integral fast flux $(\mathrm{E}>1 \mathrm{MeV})$ calculated on the entire volumes of the core barrel, the RPV and the two blocks of the concrete bio-shield - the inner and the outer parts, labelled as $\mathrm{BIO} 1$ and $\mathrm{BIO} 2$, respectively. Because of the tallied volumes in MCNP were large, the statistical errors were negligibly small. The table shows both the Pearson correlation coefficients and their $95 \%$ confidence intervals assessed using the Z-Fisher 
Table 1. Correlations between PWR FNF and the dosimetry reaction rates for the considered models.

\begin{tabular}{|c|cccc|cccc|}
\multirow{3}{*}{ Swiss PWR } & \multicolumn{4}{|c|}{ Petale-inside } & \multicolumn{4}{c|}{ Petale-outside } \\
\cline { 2 - 9 } & Rh-103 & In-115 & Ni-58 & Fe-54 & Rh-103 & In-115 & Ni-58 & Fe-54 \\
\cline { 2 - 9 } PWR_Barrel & $\mathbf{0 . 7 3}$ & $\mathbf{0 . 8 6}$ & $\mathbf{0 . 8 1}$ & $\mathbf{0 . 7 9}$ & $\mathbf{0 . 7 7}$ & $\mathbf{0 . 8 8}$ & $\mathbf{0 . 7 3}$ & $\mathbf{0 . 6 7}$ \\
& {$[0.66,0.78]$} & {$[0.83,0.89]$} & {$[0.76,0.85]$} & {$[0.74,0.83]$} & {$[0.72,0.82]$} & {$[0.83,0.89]$} & {$[0.85,0.91]$} & {$[0.76,0.85]$} \\
\hline \hline PWR_RPV & $\mathbf{0 . 5 0}$ & $\mathbf{0 . 6 2}$ & $\mathbf{0 . 5 8}$ & $\mathbf{0 . 5 7}$ & $\mathbf{0 . 5 6}$ & $\mathbf{0 . 6 9}$ & $\mathbf{0 . 6 0}$ & $\mathbf{0 . 5 5}$ \\
\hline \hline & {$[0.40,0.58]$} & $0.54,0.69]$ & {$[0.50,0.66]$} & {$[0.48,0.64]$} & {$[0.47,0.64]$} & {$[0.54,0.69]$} & {$[0.62,0.75]$} & {$[0.50,0.66]$} \\
PWR_BIO1 & $\mathbf{0 . 3 2}$ & $\mathbf{0 . 4 9}$ & $\mathbf{0 . 4 6}$ & $\mathbf{0 . 4 5}$ & $\mathbf{0 . 4 4}$ & $\mathbf{0 . 6 4}$ & $\mathbf{0 . 5 7}$ & $\mathbf{0 . 5 1}$ \\
& {$[0.20,0.42]$} & $0.39,0.58]$ & {$[0.36,0.55]$} & {$[0.34,0.54]$} & {$[0.33,0.53]$} & {$[0.39,0.58]$} & {$[0.57,0.71]$} & {$[0.36,0.55]$} \\
PWR_BIO2 & $\mathbf{0 . 3 4}$ & $\mathbf{0 . 3 5}$ & $\mathbf{0 . 4 1}$ & $\mathbf{0 . 4 1}$ & $\mathbf{0 . 4 2}$ & $\mathbf{0 . 3 9}$ & $\mathbf{0 . 5 0}$ & $\mathbf{0 . 5 0}$ \\
& {$[0.19,0.41]$} & {$[0.16,0.39]$} & {$[0.24,0.46]$} & {$[0.25,0.46]$} & {$[0.24,0.45]$} & {$[0.16,0.39]$} & {$[0.18,0.40]$} & {$[0.24,0.46]$} \\
\cline { 2 - 8 }
\end{tabular}

\begin{tabular}{|c|c|c|c|c|c|c|c|c|c|c|c|c|}
\hline \multirow[t]{2}{*}{ Swiss PWR } & \multicolumn{6}{|c|}{ HBR2-capsule } & \multicolumn{6}{|c|}{ HBR2-cavity } \\
\hline & $\mathrm{Np}-237$ & U- 238 & $\mathrm{Ni}-58$ & Fe-54 & Ti-46 & Cu-63 & Np-237 & U-238 & $\mathrm{Ni}-58$ & Fe-54 & Ti-46 & Cu-63 \\
\hline \multirow[t]{2}{*}{ PWR_Barrel } & 0.94 & 0.89 & 0.74 & 0.71 & 0.51 & 0.37 & 0.80 & 0.72 & 0.59 & 0.49 & 0.27 & 0.20 \\
\hline & {$[0.92,0.95]$} & {$[0.86,0.91]$} & {$[0.68,0.79]$} & {$[0.64,0.76]$} & {$[0.42,0.60]$} & {$[0.26,0.47]$} & {$[0.75,0.84]$} & {$[0.66,0.78]$} & {$[0.50,0.66]$} & {$[0.40,0.58]$} & {$[0.15,0.38]$} & {$[0.08,0.31$} \\
\hline PWR_RPV & 0.93 & 0.91 & 0.81 & 0.78 & 0.63 & 0.52 & 0.81 & 0.78 & 0.74 & 0.66 & 0.45 & 0.37 \\
\hline \multirow{3}{*}{ PWR_BIO1 } & {$[0.91,0.94]$} & {$[0.89,0.93]$} & {$[0.76,0.85]$} & {$[0.73,0.82]$} & {$[0.55,0.70]$} & {$[0.42,0.60]$} & {$[0.77,0.85]$} & {$[0.73,0.83]$} & {$[0.68,0.79]$} & {$[0.59,0.72]$} & {$[0.35,0.54]$} & {$[0.26,0.47]$} \\
\hline & 0.88 & 0.88 & 0.75 & 0.71 & 0.60 & 0.52 & 0.87 & 0.91 & 0.82 & 0.73 & 0.50 & 0.43 \\
\hline & {$[0.84,0.90]$} & {$[0.85,0.90]$} & {$[0.69,0.80]$} & {$[0.65,0.77]$} & {$[0.52,0.68]$} & {$[0.42,0.60]$} & {$[0.84,0.90]$} & {$[0.89,0.93]$} & {$[0.78,0.86]$} & {$[0.66,0.78]$} & {$[0.41,0.59]$} & {$[0.32,0.52]$} \\
\hline \multirow[t]{2}{*}{ PWR_BIO2 } & 0.60 & 0.65 & 0.76 & 0.76 & 0.73 & 0.66 & 0.44 & 0.48 & 0.82 & 0.83 & 0.73 & 0.66 \\
\hline & {$[0.51,0.67]$} & {$[0.57,0.72]$} & {$[0.70,0.80]$} & {$[0.70,0.80]$} & {$[0.67,0.78]$} & {$[0.59,0.72]$} & {$[0.34,0.54]$} & {$[0.38,0.57]$} & {$[0.77,0.85]$} & {$[0.79,0.87]$} & {$[0.67,0.78]$} & {$[0.58,0.72$} \\
\hline
\end{tabular}

\begin{tabular}{c|ccc|cc|cc|}
\cline { 2 - 7 } Swiss PWR & \multicolumn{7}{|c|}{ PCA-REPLICA } \\
\cline { 2 - 8 } & 1_In-115 & 1_S-32 & 2_In-115 & 2_S-32 & 3_In-115 & 3_S-32 \\
\cline { 2 - 8 } PWR_Barrel & $\mathbf{0 . 7 0}$ & $\mathbf{0 . 5 4}$ & $\mathbf{0 . 6 7}$ & $\mathbf{0 . 4 8}$ & $\mathbf{0 . 6 3}$ & $\mathbf{0 . 4 2}$ \\
& {$[0.63,0.76]$} & {$[0.45,0.62]$} & {$[0.60,0.73]$} & {$[0.38,0.57]$} & {$[0.56,0.70]$} & {$[0.32,0.52]$} \\
\hline \hline PWR_RPV & $\mathbf{0 . 8 7}$ & $\mathbf{0 . 7 8}$ & $\mathbf{0 . 8 2}$ & $\mathbf{0 . 7 4}$ & $\mathbf{0 . 7 7}$ & $\mathbf{0 . 6 8}$ \\
\hline \hline & {$[0.84,0.90]$} & {$[0.72,0.82]$} & {$[0.77,0.85]$} & {$[0.68,0.79]$} & {$[0.71,0.81]$} & {$[0.61,0.74]$} \\
PWR_BIO1 & $\mathbf{0 . 8 4}$ & $\mathbf{0 . 7 0}$ & $\mathbf{0 . 8 8}$ & $\mathbf{0 . 7 6}$ & $\mathbf{0 . 8 7}$ & $\mathbf{0 . 7 3}$ \\
& {$[0.80,0.87]$} & {$[0.63,0.76]$} & {$[0.85,0.91]$} & {$[0.70,0.81]$} & {$[0.84,0.90]$} & {$[0.67,0.78]$} \\
PWR_BIO2 & $\mathbf{0 . 5 8}$ & $\mathbf{0 . 7 7}$ & $\mathbf{0 . 4 7}$ & $\mathbf{0 . 8 5}$ & $\mathbf{0 . 4 3}$ & $\mathbf{0 . 8 3}$ \\
& {$[0.50,0.66]$} & {$[0.71,0.81]$} & {$[0.37,0.56]$} & {$[0.81,0.88]$} & {$[0.32,0.52]$} & {$[0.79,0.86]$} \\
\cline { 2 - 7 } & \multicolumn{7}{|c|}{}
\end{tabular}

transformation method (see https://cran.r-project.org/ for details).

It can be seen that the HBR2 and PCA-Replica experimental benchmarks provide reference measured data that have sufficiently high levels of correlations with the PWR RPV (and bio-shield) FNF responses. Even though this could be expected to be the case for the benchmarks dedicated for the FNF predictions validation, the correlation coefficients provide useful quantitative information on the representativity of the given benchmarks for validation assessments for the given PWR reactor. Furthermore, the correlation data is also needed for data assimilation techniques, which can be considered for a follow up work between EPFL and PSI.

Finally, as concerns the expected PETALE dosimetry measurements, the obtained results suggest that they may be less suitable for the RPV FNF validation, however they should be well applicable for validation of PWR barrel (and core shroud) FNF predictions, which is also practically relevant. This conclusion might be further verified when the uncertainties on the MCNP calculation of the PETALE models are improved (see above). This will be tacked in a future work.

\section{Discussion and conclusions}

When the neutron transport calculations are realized with modern Monte Carlo codes using detailed geometry modelling, one of the primary sources of uncertainty is the nu- clear data. Validation studies using available experimental information typically aim at qualification of the calculation methodology in conjunction with the utilized nuclear data libraries. Therefore, the measured parameters selected for validation studies should have high sensitivity to the nuclear data to be relevant. In addition, if the sensitivity is similar for the benchmark and application cases, then the code validation on the benchmark is more relevant for the target application. Such similarity is conventionally assessed using PCC. The latter can be easily derived using a stochastic sampling approach. Such assessment is demonstrated in this work in application to the LWR dosimetry studies, as an integral extension of a more traditional ND UQ evaluations [22], [23].

The provided results are preliminary because of several limitations. First, the total number of random calculations for each benchmark was relatively low, i.e. about 250. Second, the Monte Carlo statistical uncertainties for certain dosimeter reaction rates calculated with MCNP and SERPENT were relatively high, which could result in underestimated correlations. Third, the PETALE experimental design is not finalized yet and in principle can still be updated. Fourth, in order to improve the statistical precision for the dosimeter reaction rates calculations, the PETALE detector volumes were artificially and significantly increased, which is a possible source of bias. Nevertheless, analysing the presented results, one can conclude that the reported correlations obtained with the help of the NUSS tool meet the expectations dictated by the 
physical properties of the systems. For example, one can see rather high correlations between the HBR-2 and PCAReplica dosimeters to the realistic PWR FNF at the core barrel and RPV, as it should be expected. At the same time, it is interesting to see that strong correlations can be found also for the PWR concrete bio-shield zones. This information may be useful when validation of FNF at such distant locations is needed. Furthermore, one can see that the PETALE dosimeters would also be applicable for validation of FNF, e.g., at the PWR core barrel or core shroud locations.

So far the representativity of the PETALE experiments for the validation of BWR FNF evaluations was not analysed explicitly. Nevertheless, previous studies [15] already showed that in fact PWR and BWR RPV FNF simulations are highly correlated. Based on this information one can deduce that the PETALE experiments should be equally useful for validation studies of both types of LWR reactors.

Regarding future works, it can be proposed to extend the given study by including the full set of the PETALE dosimeters into the analysis (see [8]) and, as discussed, to improve the precision of the statistical evaluations and Monte Carlo calculations.

\section{Acknowledgment}

This work was partially funded by swissnuclear through the PSI/SN R\&D project LRT-01 (2018-2019). The authors express their sincere gratitude to Dr. Mathieu Hursin for his assistance with SERPENT simulations and valuable discussions, as well as to Dr. Erwin Alhassan for his kind contribution to the paper preparation.

\section{References}

[1] A.Vasiliev, H. Ferroukhi, M.A. Zimmermann, R. Chawla, Ann. Nucl. Energy 34, 615-627 (2007).

[2] A. Vasiliev, W. Wieselquist, H. Ferroukhi, S. Canepa, J. Heldt, G. Ledergerber, Progress in Nucl. Sci. and Technol. 4, 99-103 (2014).

[3] A. Dupré, A. Vasiliev, H. Ferroukhi, A. Pautz, Ann. Nucl. Energy 85, 820-829 (2015).

[4] L.S. Waters (Ed.), LANL, LA-CP-02-408 (2002).

[5] M.B. Chadwick, et. al., Nucl. Data Sheets 107, 29313060 (2006).
[6] V. Lamirand, G. Perret, S. Radman, D. Siefman, P. Frajtag, M. Hursin, A. Gruel, P. Leconte, P. Blaise, and A. Pautz, Proc. 16th Int. Symp. Reactor Dosimetry, ASTM STP1608, ASTM International, West Conshohocken, PA (2018).

[7] V. Lamirand, A. Laureau, D. Rochman, G. Perret, A. Gruel, P. Leconte, P. Blaise, and A. Pautz, EPJ Web of Conferences 211, 03003 (2019).

[8] A. Laureau, V. Lamirand, D. Rochman, and A. Pautz, Proc. Int. Conf. ND2019 (2019).

[9] W. Wieselquist, T. Zhu, A. Vasiliev, H. Ferroukhi, Sci. Technol. Nucl. Ins. 2013, Article ID 549793, 1-15 (2013).

[10] T. Zhu, A. Vasiliev, W. Wieselquist, H. Ferroukhi, Proc. Int. Conf. Physor-2012 (2012).

[11] T. Zhu, A. Vasiliev, H. Ferroukhi, D. Rochman, A. Pautz, Nucl. Sci. Eng. 184, 69-83 (2016).

[12] A. Vasiliev, J. Herrero, M. Pecchia, D. Rochman, H. Ferroukhi, S. Caruso, Materials 201912 494, 1-30 (2019).

[13] T. Zhu, A. Vasiliev, H. Ferroukhi, A. Pautz, Ann. Nucl. Energy 75, 713-722 (2015).

[14] C.M. Perfetti and B.T. Rearden, Nucl. Sci. Eng. 182, 354-368 (2016).

[15] A. Vasiliev, D. Rochman, M. Pecchia, H. Ferroukhi, Energies 2016, 9 1039, 1-18 (2016).

[16] A. Vasiliev, J. Herrero, D. Rochman, M. Pecchia, H. Ferroukhi, S. Caruso, Proc. Int. Conf. BEPU-2018 (2018).

[17] A. Vasiliev, D. Rochman, M. Pecchia, H. Ferroukhi, Ann. Nucl. Energy 116, 57-68 (2018).

[18] R. Pittarello, A. Vasiliev, H. Ferroukhi, R. Chawla, Ann. Nucl. Energy 38, 1842-1851 (2011).

[19] A. Vasiliev, H. Ferroukhi, E. Kolbe, Ann. Nucl. Energy 37, 1404-1410 (2010).

[20] SINBAD-PCA-REPLICA, NEA-1517/94 (2017).

[21] H. Sjöstrand, N. Asquith, P. Helgesson, D. Rochman, and S. van der Marck, EPJ Nuclear Sci. Technol. 4, 15 (2018).

[22] A. Vasiliev, H. Ferroukhi, T. Zhu, A. Pautz, Nucl. Data Sheets 118, 575-578 (2014).

[23] A. Vasiliev, M. Pecchia, D. Rochman, H. Ferroukhi, E. Alhassan, Proc. Int. Conf. ND2019 (2019). 